\title{
Phenotyping of CYP450 in human liver microsomes using the cocktail approach
}

\author{
Dany Spaggiari • Laurent Geiser • Youssef Daali • \\ Serge Rudaz
}

Received: 28 February 2014 / Revised: 7 May 2014 / Accepted: 19 May 2014 /Published online: 4 June 2014

(C) Springer-Verlag Berlin Heidelberg 2014

\begin{abstract}
The cocktail approach is an advantageous strategy used to monitor the activities of several cytochromes P450 (CYPs) in a single test to increase the throughput of in vitro phenotyping studies. In this study, a cocktail mixture was developed with eight CYP-specific probe substrates to simultaneously evaluate the activity of the most important CYPs, namely, CYP1A2, CYP2A6, CYP2B6, CYP2C9, CYP2C19, CYP2D6, CYP2E1, and the CYP3A subfamily. After cocktail incubation in the presence of human liver microsomes (HLMs), the eight selected substrates and their specific metabolites were analyzed by ultra-high-pressure liquid chromatography and electrospray ionization quadrupole time-offlight mass spectrometry. Qualitative and quantitative data were simultaneously acquired to produce an overview of the extended phase I biotransformation routes for each probe substrate in the HLMs and to generate phenotypic profiles of various HLMs. A comparison of the cocktail strategy with an individual substrate assay for each CYP produced similar results. Moreover, the cocktail was tested on HLMs with different allelic variants and/or in the presence of selective inhibitors. The results were in agreement with the genetic polymorphisms of the CYPs and the expected effect of the alterations. All of these experiments confirmed the reliability of this cocktail assay for phenotyping of the microsomal CYPs.
\end{abstract}

D. Spaggiari $\cdot$ L. Geiser $\cdot$ Y. Daali $\cdot$ S. Rudaz $(\varangle)$

School of Pharmaceutical Sciences, University of Geneva, University of Lausanne, Boulevard d'Yvoy 20, 1211 Geneva 4, Switzerland

e-mail: serge.rudaz@unige.ch

Y. Daali

Division of Clinical Pharmacology and Toxicology,

Geneva University Hospitals, Rue Gabrielle-Perret-Gentil 4,

1211 Geneva 14, Switzerland
Keywords Cocktail approach · Ultra-high-pressure liquid chromatography-quadrupole time-of-flight mass spectrometry · Cytochrome P450 profile · Phenotyping · Genetic polymorphism $\cdot$ Human liver microsomes

\section{Introduction}

Cytochromes P450 (CYPs) are the major phase I metabolic enzymes involved in the biotransformation of xenobiotics and endogenous compounds [1]. The activity of CYPs is characterized by a high interindividual variability due to environmental factors (e.g., diet, drug therapy, toxic substances) and/or genetic polymorphisms, which are part of the individual CYP phenotype. Certain major isoforms of the CYP superfamily involved in the metabolism of marketed drugs- CYP2C9, CYP2C19, and CYP2D6 - are recognized as highly polymorphic. According to the type of allelic variant affecting these CYPs, genetic polymorphism can significantly alter their metabolic activity, thus modifying the clinical response and/or increasing the risk of drug-drug interactions (DDI). The evaluation of genetic polymorphism can be performed by sequencing the DNA of the CYP genes (genotyping) or by determining the CYP expression/activity (phenotyping). In certain cases, owing to the genetic and epigenetic regulations, the relationship between the CYP gene and the enzymatic activity is not trivial, which makes the test selection (e.g., constitutional or functional) for predicting the drug's clinical response difficult. However, the evaluation of the DDI is possible only by CYP phenotyping [2].

Currently, in vitro phenotyping of CYPs has been successfully used to enhance the background understanding of the behavior of polymorphic CYPs involved in xenobiotic metabolism and DDI. For this purpose, human liver microsomes (HLMs) are typically used as the in vitro native phase I enzyme source for metabolism studies owing to their simple preparation and ease of handling and the commercial availability of 
numerous allelic variants. New strategies for performing in vitro metabolism studies have been developed recently with a particular focus on inexpensive automation and reduction of reagent consumption [3, 4]. High-throughput strategies for microsomal CYP investigations based on liquid chromatography (LC)-mass spectrometry (MS) have been developed with regard to its powerful separation capabilities, including high sensitivity, selectivity, and resolution [5, 6]. Among these strategies, the cocktail approach has been developed recently to efficiently and rapidly monitor the activities of several CYPs within a single test. In this study, selective CYP substrates, called probes, are incubated with microsomes, consecutively reducing the time and assay costs. Several in vitro cocktail approaches have been reported in the literature with various numbers of probe substrates and analytical strategies. The current status of cocktail approaches and methods to investigate CYP activities in HLMs has been reviewed recently [7]. In the context of phenotyping of CYPs, the use of a highresolution device, such as a quadrupole time-of-flight (QTOF) mass spectrometer, is appealing considering that one CYP is often involved in the formation of numerous metabolites. With this type of instrumentation, untargeted acquisition provides an extended phenotypic profile of the CYPs. Hence, the substrates and their expected and unexpected metabolites are sensitively and accurately detected and identified within a single run. An additional advantage of this instrumentation is that data can be handled, processed, and filtered after the acquisition with specific commercially available software for untargeted metabolite detection and/ or identification. Therefore, the QTOF instrument could present several benefits for in vitro CYP phenotyping investigations.

In this study, a cocktail assay was originally designed for the phenotyping of the most important CYPs in humans, namely, CYP1A2, CYP2A6, CYP2B6, CYP2C9, CYP2C19, CYP2D6, CYP2E1, and the CYP3A subfamily. An LC-MS ${ }^{\mathrm{E}}$ method using generic gradient conditions was developed on an ultra-highpressure LC (UHPLC)-QTOF platform for the reliable quantitative and qualitative analysis of this cocktail metabolization. The CYP activity assessment was based on the metabolic ratio. A radar plot representation of the CYP phenotypic profile was proposed for a better comparison of the different assays and the various phenotypes determined. A comparison was made between the cocktail approach and classic tests, which perform individual incubations of the substrates. The latter was then evaluated for the CYP phenotyping of allelic variant HLMs. An altered allelic variant HLM was finally evaluated by addition of CYP-specific inhibitors and human recombinant enzymes.

\section{Materials and methods}

Chemicals, reagents, test compounds, and other materials

Methanol (MeOH), acetonitrile (ACN), and water of ultra LCMS grade were purchased from Biosolve (Valkenswaard,
Netherlands). Formic acid (98-100 \%) was obtained from Merck (Darmstadt, Germany). Chlorzoxazone (98\%), 6hydroxychlorzoxazone (97\%), $( \pm)-4^{\prime}$-hydroxymephenytoin (98\%), 4'-hydroxyflurbiprofen (98\%), and hydroxybupropion (95\%) were purchased from Toronto Research Chemicals (Toronto ON, Canada). Potassium hydroxide, 4-(2hydroxyethyl)piperazine-1-ethanesulfonic acid sodium salt (HEPES) (99.5\%), dextromethorphan hydrobromide (99\%), dextrorphan tartrate (98\%), bupropion hydrochloride (98\%), phenacetin (97\%), acetaminophen (99\%), flurbiprofen (99\%), coumarin (99\%), 7-hydroxycoumarin (99\%), ketoconazole (98\%), and anhydrous quinidine (98\%) were obtained from Sigma-Aldrich (Buchs, Switzerland), whereas methanolic stock solutions of midazolam and 1-hydroxymidazolam were purchased from Lipomed (Arlesheim, Switzerland). (S)Mephenytoin (99 \%) was obtained from Enzo Life Sciences (Lausen, Switzerland), reduced $\beta$-nicotinamide adenine dinucleotide 2'-phosphate tetrasodium salt (NADPH) $(96 \%)$ was obtained from Applichem (Darmstadt, Germany), and leucineenkephalin was obtained from Bachem (Bubendorf, Switzerland). Pooled HLMs from 30 donors (i.e., reference HLMs) and HLMs with allelic variants $\left(C Y P 2 D 6^{*} 4 / * 4, C Y P 2 C 9^{*} 3 / * 3\right.$, and CYP2C19*2/2) from single donors (HH35, HH519, and HH689, respectively) were purchased from BD Biosciences (Allschwil, Switzerland). Human CYP Baculosomes reagent (baculosome CYP2D6) was obtained from Invitrogen (Basel, Switzerland). Aliquots of HLMs and baculosome CYP2D6 were stored at $-80^{\circ} \mathrm{C}$. A $50 \mathrm{mM} \mathrm{NADPH}$ stock solution was prepared in water and stored at $-20{ }^{\circ} \mathrm{C}$. Immediately before the CYPdependent metabolism assays, intermediate concentrations of the HLMs and baculosome CYP2D6 were prepared by diluting aliquots with the $50 \mathrm{mM}$ HEPES (pH 7.4) buffer solution, which was prepared by dissolving the required amount of HEPES in water. The $\mathrm{pH}$ was adjusted to 7.4 with potassium hydroxide using a SevenMulti S40 pH meter (Mettler Toledo, Greifensee, Switzerland). The substrate, metabolite, and inhibitor stock solutions at $1 \mathrm{mg} / \mathrm{mL}$ were prepared in $\mathrm{MeOH}$ and stored at $-20^{\circ} \mathrm{C}$ for 6 months or less. Intermediate solutions were appropriately reconstituted daily in the $50 \mathrm{mM}$ HEPES (pH 7.4) buffer solution.

\section{Characterization of CYP-dependent metabolism in HLMs}

\section{Incubation method}

All of the microsomal incubations were performed in duplicate in a final incubation volume of $100 \mu \mathrm{L}$. The reaction medium contained $50 \mathrm{mM}$ HEPES ( $\mathrm{pH}$ 7.4), excess NADPH, $0.5 \mathrm{mg}$ protein per milliliter of HLMs, and an individual CYP probe substrate or a cocktail of substrates. The final substrate concentrations were obtained by adding the required volume of the intermediate substrate or cocktail solution to the reaction medium. These concentrations were as follows: $50 \mu \mathrm{M}$ for phenacetin, $2 \mu \mathrm{M}$ for coumarin, $5 \mu \mathrm{M}$ for bupropion, 
$5 \mu \mathrm{M}$ for flurbiprofen, $100 \mu \mathrm{M}$ for $(S)$-mephenytoin, $5 \mu \mathrm{M}$ for dextromethorphan, $40 \mu \mathrm{M}$ for chlorzoxazone, and $2.5 \mu \mathrm{M}$ for midazolam. The final organic solvent $(\mathrm{MeOH})$ concentration was $0.5 \%(\mathrm{v} / \mathrm{v})$. After preincubation for $3 \mathrm{~min}$ at $37^{\circ} \mathrm{C}$, the CYP-dependent phase I reactions were initiated by adding excess NADPH $(2.0 \mathrm{mM})$. The incubation proceeded for $20 \mathrm{~min}$ at $37{ }^{\circ} \mathrm{C}$ under agitation $(400 \mathrm{rpm})$. The enzymatic reaction was stopped by adding $100 \mu \mathrm{L}$ of ice-cold $\mathrm{ACN}$ to the reaction medium. The precipitated proteins were removed by centrifugation ( $5 \mathrm{~min}$ at $10,000 \mathrm{rpm}$ ), and an aliquot $(150 \mu \mathrm{L})$ of the resulting supernatant was transferred to a vial for LC-MS analysis.

Well-characterized CYP competitive inhibitors, such as quinidine (CYP2D6) and ketoconazole (CYP3A), were added at a single concentration of $1 \mu \mathrm{M}$, whereas baculosome CYP2D6 was tested at $3 \mathrm{nM}$. Each reagent was incubated in duplicate with the cocktail of substrates and the allelic variant $C Y P 2 D 6^{*} 4 / 4$ HLMs under the previously described incubation conditions.

\section{Data treatment}

Prism version 5.01 (GraphPad Software, San Diego, CA, USA) was used to determine the most appropriate fitting model for the depletion of the eight substrates (one phase decay) and the formation of the eight metabolites (one site total).

\section{Liquid chromatography-mass spectrometry}

\section{UHPLC-QTOF instrumentation}

The chromatographic analyses were performed with an Acquity UPLC system (Waters, Milford, MA, USA) able to deliver mobile phases at a pressure of up to 1,000 bar. The equipment includes a binary solvent manager with a maximum delivery flow rate of $2 \mathrm{~mL} / \mathrm{min}$, an autosampler with a $2-\mu \mathrm{L}$ loop operating in full-loop injection mode, and a column manager composed of a precolumn eluent heater and a column oven set to $40{ }^{\circ} \mathrm{C}$. The UPLC system was coupled in an optimized configuration with a Xevo ${ }^{\circledR}$ QTOF mass spectrometer (Waters, Milford, MA, USA) fitted with a Z-spray electrospray ionization (ESI) source [8]. The samples were stored at $4{ }^{\circ} \mathrm{C}$ in the autosampler prior to and during the analysis. Data acquisition, data handling, and instrument control were performed by MassLynx version 4.1 (Waters, Milford, MA, USA).

\section{Reversed-phase LC conditions}

The reversed-phase LC separations were performed on a Waters $100 \mathrm{~mm} \times 2.1 \mathrm{~mm}$ XBridge $^{\mathrm{TM}} \mathrm{BEH} \mathrm{C}_{18} \mathrm{XP}$ column with $2.5-\mu \mathrm{m}$ particle size and a flow rate of $400 \mu \mathrm{L} / \mathrm{min}$ in gradient mode (solvent $\mathrm{A}$, water with $0.1 \%$ formic acid; solvent B, ACN with $0.1 \%$ formic acid, $2-75 \%$ solvent B in $15 \mathrm{~min}$, up to $95 \%$ solvent $\mathrm{B}$ in $0.2 \mathrm{~min}$, held at $95 \%$ solvent $\mathrm{B}$ to $0.5 \mathrm{~min}$, then column reconditioning at $2 \%$ solvent $\mathrm{B}$ to $18 \mathrm{~min}$ ), with the eluate from the first $0.5 \mathrm{~min}$ diverted to waste.

\section{ESI-QTOF-MS ${ }^{E}$ parameters}

Regarding the MS operating conditions, the desolvation gas (nitrogen) flow was set to $1,000 \mathrm{~L} / \mathrm{h}$ with a temperature of $500{ }^{\circ} \mathrm{C}$, the source temperature was $150{ }^{\circ} \mathrm{C}$, the cone gas (nitrogen) flow rate was $20 \mathrm{~L} / \mathrm{h}$, the collision gas (argon) flow rate was $0.2 \mathrm{~mL} / \mathrm{min}$, and the capillary voltage was $3.0 \mathrm{kV}$ in the positive mode and $2.4 \mathrm{kV}$ in the negative mode. The cone voltage and the extraction cone voltage were similar in both positive and negative mode at $30 \mathrm{~V}$ and $4.1 \mathrm{~V}$, respectively. The microchannel plates were operated at $2,200 \mathrm{~V}$ in positive mode and at $1,750 \mathrm{~V}$ in negative mode, with a 4$\mathrm{GHz}$ time-to-digital converter. The QTOF-MS system was operated in wide-pass quadrupole mode with a low collision energy set to $4 \mathrm{eV}$ to acquire MS information in the first function, and a collision energy ramp from 20 to $35 \mathrm{eV}$ was applied in the second function $\left(\mathrm{MS}^{\mathrm{E}}\right.$ mode). The data were collected in the V-optics centroid mode over an $\mathrm{m} / \mathrm{z}$ range of $100-1,000$ for both functions with an accumulation time of $0.2 \mathrm{~s}$. The data were acquired using programmed dynamic range enhancement (pDRE) technology and an independent reference lock-mass ion infused through the LockSpray ${ }^{\mathrm{TM}}$ interface to ensure accuracy and to decrease acquisition variability. A solution of leucine-enkephalin $(\mathrm{m} / \mathrm{z}$ 556.2771 ) at a concentration of $0.2 \mu \mathrm{g} / \mathrm{mL}$ in $1: 1$ (v/v) ACN/water plus $0.1 \%$ formic acid was used as the reference compound and was infused at a flow rate of $10 \mu \mathrm{L} / \mathrm{min}$. The LockSpray frequency was set to $20 \mathrm{~s}$ (scan duration of $1 \mathrm{~s}$ ) and the data were averaged over five spectra.

Quantitative evaluation of the method

\section{Selectivity}

Method selectivity was assessed by injecting (1) HEPES buffer, (2) the analyte and cofactor in HEPES buffer, (3) a blank microsomal incubation medium with the cofactor, (4) the analyte in blank microsomal incubation medium without the cofactor, and (5) a mixture of analytes in microsomal incubation medium without the cofactor. Extracted ion chromatograms for each analyte were used to assess potential interference at the corresponding retention time. 


\section{Response function linearity}

The response function for the eight substrates and their metabolites $(N=16)$ was evaluated using microsomal incubation mixtures (without cofactor) reconstituted at concentrations ranging from 1 to $5,000 \mathrm{ng} / \mathrm{mL}$. To evaluate the response function of each specific transition, each analyte in ten concentrations $(k=10)$ injected in triplicate was investigated. A conventional least-squares linear regression without weighting was fitted to the data obtained, and the response function range was evaluated with a determination coefficient $\left(R^{2}\right)$ higher than 0.95 .

\section{Limit of detection and lower limit of quantification}

The limit of detection and the lower limit of quantification were measured in reconstituted incubation samples as the concentrations possessing a response with a signal-to-noise ratio greater than or equal to 3 and 10 , respectively.

\section{Matrix effect}

The matrix effect was determined according to method described by Matuszeski et al. [9]. Briefly, the signal intensity of the sample including the mixture of eight substrates and their metabolites added at three different concentrations (low, $50 \mathrm{ppb}$; medium, $200 \mathrm{ppb}$; high, $500 \mathrm{ppb)}$ to reconstituted incubation mixture was compared with that of the standard mixture solution.

\section{Precision and stability}

The precision of the analytical method was measured by triplicate injection of reconstituted microsomal mixtures of substrates and metabolites on different analysis days. The eight substrate and metabolite pairs were evaluated at half concentration of the substrate during incubation. The relative standard deviation obtained on the absolute peak area and the quadratic mean (or root mean square) were used for assessment of within-day and between-day variabilities, respectively. The stability of the overall bioanalytical process was evaluated by testing different aliquots of pooled HLMs on different days.

\section{Results and discussion}

Selection of substrates and evaluation of the reaction conditions

The eight CYP probe substrates of the cocktail, namely, phenacetin (CYP1A2), coumarin (CYP2A6), bupropion
(CYP2B6), flurbiprofen (CYP2C9), (S)-mephenytoin (CYP2C19), dextromethorphan (CYP2D6), chlorzoxazone (CYP2E1), and midazolam (CYP3A subfamily), were selected to monitor simultaneously the metabolic activities of clinically and toxicologically relevant cytochromes in humans. In preliminary experiments, several well-characterized probes were tested for an optimal cocktail assay. These eight compounds were finally selected according to the following: (1) in vitro recommendations based on the specificity of the CYP probe reaction (e.g., coumarin, bupropion, dextromethorphan, chlorzoxazone, and midazolam) [10], (2) in vivo and in vitro considerations (e.g., flurbiprofen), and (3) analytical and metabolic issues. Phenacetin for example, was preferred over caffeine owing to its higher microsomal metabolic rate at a low concentration, $(S)$-mephenytoin was retained for the CYP2C19 activity assessment because omeprazole was found to be degraded in acidic solution, and flurbiprofen was retained over the commoner $\mathrm{CYP} 2 \mathrm{C} 9$ in vitro probes, such as diclofenac and tolbutamide, owing to its presence in a wellknown cocktail currently used in vivo in the clinical environment $[11,12]$. Although certain authors have proposed a twosite binding model for CYP3A activity and have recommended the use of two structurally unrelated probes [10], only midazolam was included according to recent studies demonstrating the evaluation of the in vitro CYP3A subfamily activity, which was independent of the type and number of substrates [13, 14].

The adjustment of the experimental conditions for the cocktail, especially the incubation time and protein concentration, was performed with the reference HLMs (pooled). These incubation parameters were adjusted according to practical recommendations for the following reasons: (1) to avoid excessive substrate depletion (less than $20 \%$ ) and (2) to maintain a linear relationship between the amount of enzyme or the incubation time and product formation $[15,16]$. These adjustments are challenging because the concentrations of the individual CYPs in HLMs cannot be increased or decreased separately because a constant ratio is maintained among the different isoenzymes. When the reaction adjustment is based on CYPs with low abundance in HLMs (e.g., CYP1A2, CYP2C19, and CYP2D6), a higher protein concentration is required to generate a detectable amount of the metabolite, but this produces higher concentrations of the high-abundance CYPs (e.g., CYP3A4 and CYP2C9), which negatively influence the kinetics from a linear to a nonlinear behavior. An additional issue of higher microsomal protein concentrations concerns the nonspecific binding to proteins and lipids (matrix material) that can affect the reliability of the data [17]. In this study, the reaction conditions, including the enzyme protein concentration of $0.5 \mathrm{mg} / \mathrm{mL}$ and the incubation time of $20 \mathrm{~min}$, were finally selected relative to the probe substrate that presents the slowest turnover, $(S)$-mephenytoin (CYP2C19). It has been shown elsewhere that the latter 
requires a longer incubation and a higher protein concentration to generate sufficient metabolite for detection [18]. As depicted in Fig. 1, under these experimental conditions, the in vitro recommendations were followed. As expected, substrates (e.g., midazolam and coumarin) with a rapid turnover and low Michaelis-Menten constants $\left(K_{\mathrm{m}}\right)$ were highly depleted, and their major metabolites were primarily formed after $20 \mathrm{~min}$. This incubation time exceeded the optimal incubation time (15 min or less) to maintain the linearity of 1'-hydroxymidazolam and 7-hydroxycoumarin production $[19,20]$. Linear metabolite formation and low substrate disappearance were followed for the other CYPs monitored. Finally, these kinetic conditions were considered as acceptable because the linear metabolite formation was followed for the most of the CYPs, including the highly polymorphic CYPs (CYP2C9, CYP2C19, and CYP2D6).

The concentrations of seven of the eight substrates were set near or below their respective $K_{\mathrm{m}}$ values (Table 1) for the following reasons: (1) to maintain the high specificity of the probe reaction, (2) to avoid or reduce potential interactions among the substrates of the cocktail, and (3) to minimize the percentage of organic solvent generally used for better substrate solubility but that could significantly modify the CYPmediated metabolism [22]. Only the slow turnover probe $(S)$ mephenytoin required its inclusion in the cocktail at a concentration higher than its $K_{\mathrm{m}}$ to detect its metabolite. Preliminary probe-probe interaction experiments showed that bupropion (probe substrate for CYP2B6) interfered with the activity of several CYPs, in particular that of CYP2C19. Therefore, the concentration of bupropion was decreased to less than $1 / 15$ of the initial value. Hence, the observed interaction between this substrate and this isoform was reduced [a slight increase of CYP2C19-mediated ( \pm - $-4^{\prime}$ hydroxymephenytoin production], as reported elsewhere [20].

\section{LC-MS ${ }^{\mathrm{E}}$ method}

Considering the heterogeneous physicochemical properties of the analytes studied, a generic gradient-based analytical reversed-phase LC method was used to monitor the activities of the eight CYP450 isoforms. With the cocktail developed, the eight CYP probe substrates and their metabolites were simultaneously analyzed in microsomal incubations, and are listed in Table 1 along with the exact mass $(\mathrm{m} / \mathrm{z})$ and polarity of the ESI used for the detection. The initial experiments were performed to evaluate and optimize the ESI-MS response by using both the positive mode and the negative mode and by varying the cone voltages (fixed or ramped). Most of the compounds showed good sensitivity in positive ionization mode, except for chlorzoxazone/6-hydroxychlorzoxazone and flurbiprofen/4'-hydroxyflurbiprofen, with sensitivities that were significantly better in negative ionization mode. In terms of absolute sensitivity (i.e., ion counts), the optimal cone voltage was $30 \mathrm{~V}$, regardless of the polarity chosen. As depicted in Fig. 2, by use of a generic gradient LC separation, all of the analytes were eluted with retention times ranging
Fig. 1 Depletion of probe substrates and formation of cytochrome P450 (CYP)-specific metabolites in human liver microsomes (HLMs) at $0.5 \mathrm{mg}$ protein per milliliter as a function of time. Substrate depletion is expressed as a percentage of the amount of substrate at $t=0 \mathrm{~min}$, and metabolite formation is expressed as a percentage of the amount of metabolite at $t=$ $120 \mathrm{~min}$
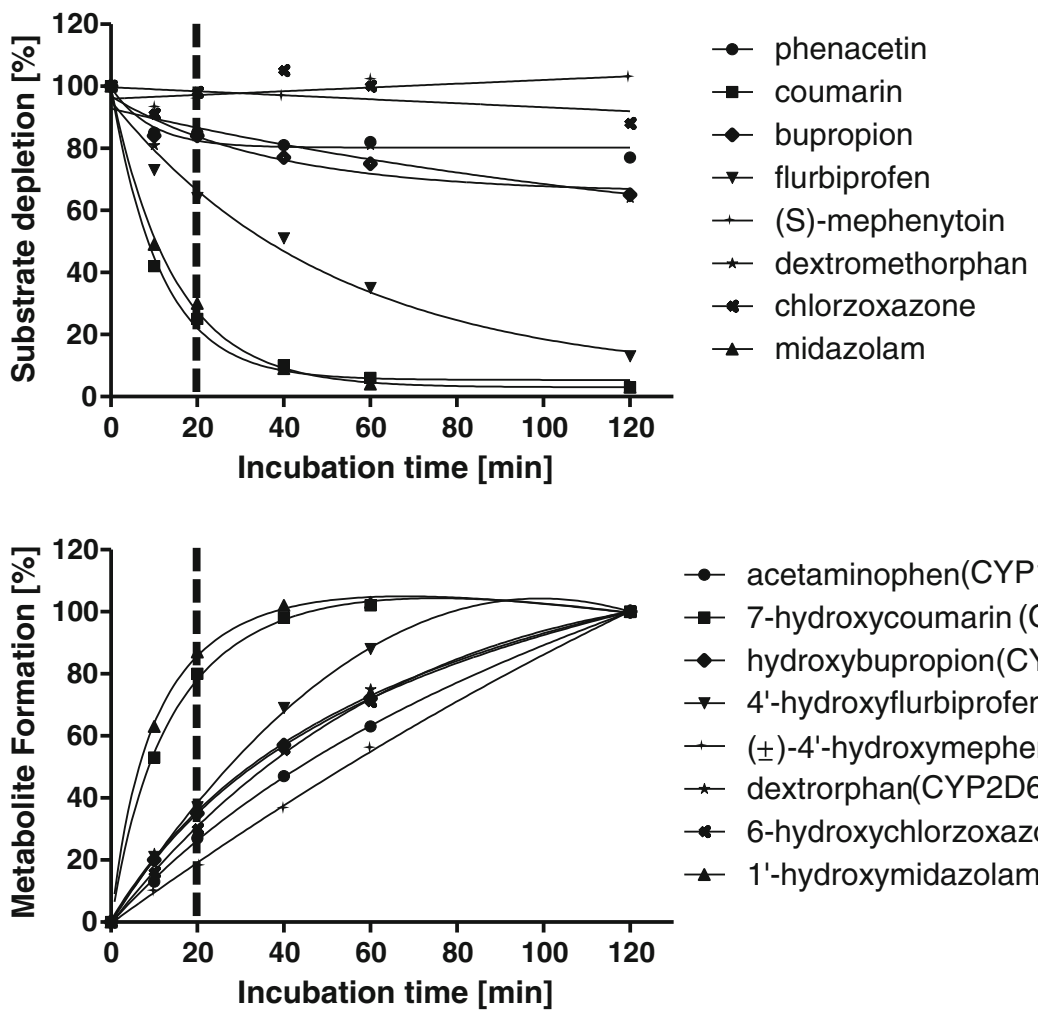

$\rightarrow$ acetaminophen(CYP1A2)

$\rightarrow$ 7-hydroxycoumarin (CYP2A6)

$\rightarrow$ hydroxybupropion(CYP2B6)

$\rightarrow \quad$ 4'-hydroxyflurbiprofen(CYP2C9)

$\leftarrow$ ( \pm )-4'-hydroxymephenytoin(CYP2C19)

* dextrorphan(CYP2D6)

- 6-hydroxychlorzoxazone(CYP2E1)

$\_$1'-hydroxymidazolam(CYP3A) Incubation time [min] 
Table 1 The eight substrates selected for the cocktail approach, with their cytochrome P450 (CYP)-specific metabolites and exact detection mass

\begin{tabular}{|c|c|c|c|c|c|c|c|}
\hline CYP isoform & Probe substrate & $\begin{array}{l}\text { CYP-specific } \\
\text { reaction }\end{array}$ & CYP-specific metabolite & $\begin{array}{l}K_{\mathrm{m}}^{\mathrm{a}} \\
(\mu \mathrm{M})\end{array}$ & $\begin{array}{l}\text { Concentration for } \\
\text { incubation }(\mu \mathrm{M})\end{array}$ & $\begin{array}{l}\text { Exact mass } \\
(\mathrm{m} / \mathrm{z})\end{array}$ & Polarity \\
\hline \multirow[t]{2}{*}{$1 \mathrm{~A} 2$} & \multirow[t]{2}{*}{ Phenacetin } & \multirow[t]{2}{*}{ O-Deethylation } & & $1.7-152$ & 50 & 180.1024 & $\mathrm{ES}^{+}$ \\
\hline & & & Acetaminophen & & & 152.0675 & $\mathrm{ES}^{+}$ \\
\hline \multirow[t]{2}{*}{$2 \mathrm{~A} 6$} & \multirow[t]{2}{*}{ Coumarin } & \multirow[t]{2}{*}{ 7-Hydroxylation } & & $0.3-2.3$ & 2 & 147.0446 & $\mathrm{ES}^{+}$ \\
\hline & & & 7-Hydroxycoumarin & & & 163.0395 & $\mathrm{ES}^{+}$ \\
\hline \multirow[t]{2}{*}{ 2B6 } & \multirow[t]{2}{*}{ Bupropion } & \multirow[t]{2}{*}{ Hydroxylation } & & $67-168$ & 5 & 240.1155 & $\mathrm{ES}^{+}$ \\
\hline & & & Hydroxybupropion & & & $238.0998\left(-\mathrm{H}_{2} \mathrm{O}\right)$ & $\mathrm{ES}^{+}$ \\
\hline \multirow[t]{2}{*}{$2 \mathrm{C} 9$} & \multirow[t]{2}{*}{ Flurbiprofen } & \multirow[t]{2}{*}{ 4'-Hydroxylation } & & $6-42$ & 5 & $199.0923(-\mathrm{COO})$ & $\mathrm{ES}^{-}$ \\
\hline & & & 4'-Hydroxyflurbiprofen & & & $215.0875(-\mathrm{COO})$ & $\mathrm{ES}^{-}$ \\
\hline \multirow[t]{2}{*}{$2 \mathrm{C} 19$} & \multirow[t]{2}{*}{$(S)$-Mephenytoin } & \multirow[t]{2}{*}{ 4'-Hydroxylation } & & $13-35$ & 100 & 219.1134 & $\mathrm{ES}^{+}$ \\
\hline & & & $( \pm)-4^{\prime}-$ Hydroxymephenytoin & & & 235.1083 & $\mathrm{ES}^{+}$ \\
\hline \multirow[t]{2}{*}{ 2D6 } & \multirow[t]{2}{*}{ Dextromethorphan } & \multirow[t]{2}{*}{ O-Demethylation } & & $0.4-8.5$ & 5 & 272.2014 & $\mathrm{ES}^{+}$ \\
\hline & & & Dextrorphan & & & 258.1858 & $\mathrm{ES}^{+}$ \\
\hline \multirow[t]{2}{*}{$2 \mathrm{E} 1$} & \multirow[t]{2}{*}{ Chlorzoxazone } & \multirow[t]{2}{*}{ 6-Hydroxylation } & & $39-157$ & 40 & 167.9852 & $\mathrm{ES}^{-}$ \\
\hline & & & 6-Hydroxychlorzoxazone & & & 183.9801 & $\mathrm{ES}^{-}$ \\
\hline \multirow[t]{2}{*}{ 3A subfamily } & \multirow[t]{2}{*}{ Midazolam } & \multirow[t]{2}{*}{ 1'-Hydroxylation } & & $1-14$ & 2.5 & 326.0860 & $\mathrm{ES}^{+}$ \\
\hline & & & 1'-Hydroxymidazolam & & & 342.0809 & $\mathrm{ES}^{+}$ \\
\hline
\end{tabular}

ES electrospray

${ }^{\mathrm{a}}$ From [21]

from 2 to $10.5 \mathrm{~min}$ and were separated using the accurate mass feature of the mass spectrometer $( \pm 0.02 \mathrm{Da}$, first function MS). With use of the selected gradient, the substrate/ metabolite ion suppression effects were avoided because each substrate was eluted after its respective metabolites. Low chromatographic selectivity between midazolam and its metabolite, 1'-hydroxymidazolam, was observed, along with certain critical selectivity windows for $(S)$-mephenytoin/dextromethorphan, phenacetin/coumarin/bupropion, and 7hydroxycoumarin/dextrorphan. However, because of the MS dimensions, an overall resolution was obtained. To extract the maximum information from the microsomal metabolism, a multiresidue method that allows both quantitative and qualitative $\left(\mathrm{MS}^{\mathrm{E}}\right)$ analysis was considered. Compared with a classic time-of-flight MS acquisition, this detection mode consisted of a first function (quantitative data) obtained in the wide-pass quadrupole mode at a low fixed collision energy $(4 \mathrm{eV})$ and an alternated second function (qualitative data) with a collision energy ramping from 20 to $35 \mathrm{eV}$. Combining the MS/MS spectra obtained and postprocessing of the data with automated software, one can reveal the complementary metabolites that can potentially be formed.

Furthermore, in metabolism studies performed with the cocktail substrates, the analytes are present in the incubation sample over a wide range of concentrations. To overcome this highly dynamic concentration range issue, which could lead to incorrect exact mass measurements owing to the saturation of the time-to-digital converter detector, a special feature, pDRE, should be activated. In this case, an unattenuated and an attenuated accumulation time are combined within the run to generate a stitched data point where the measured mass could be considered as exact. The combination of $\mathrm{MS}^{\mathrm{E}}$ and $\mathrm{pDRE}$ significantly decreased the effective data acquisition rate of the time-of-flight MS analyzer. With an accumulation time of $0.2 \mathrm{~s}(5 \mathrm{~Hz}$, excluding the interscan delay), the effective acquisition rate corresponded to approximately $0.8 \mathrm{~s}$ $(1.25 \mathrm{~Hz})$. This value is not compatible with the very thin peaks obtained with the narrow-bore column packed with sub$2-\mu \mathrm{m}$ particles. Considering the effective acquisition rate, sufficient data acquisition points (three points for each function) to achieve good quantitative data and MS data of sufficient quality for reprocessing could not be obtained under the optimal UHPLC conditions $(0.04 \mathrm{~min}$ at $4.4 \%$ of the peak height at $600 \mu \mathrm{L} / \mathrm{min}$ ). To address this constraint, a $100-\mathrm{mm}$ column packed with larger particles $(2.5 \mu \mathrm{m})$ was used instead of a shorter column $(50 \mathrm{~mm})$ packed with smaller particles (1.7- $\mu \mathrm{m}$ particle inner diameter). With use of this configuration, the following advantages were obtained: (1) the peak width adapted to the data acquisition rate $(0.11 \mathrm{~min}$ at $4.4 \%$ of the peak height at $400 \mu \mathrm{L} / \mathrm{min}$ ), (2) equivalent chromatographic performance (equivalent column length to particle size ratio), (3) reduced extra-column broadening (higher column variance), (4) reduced backpressure (380 bar, which corresponds to a decrease of approximately $40 \%$ ), and (5) 


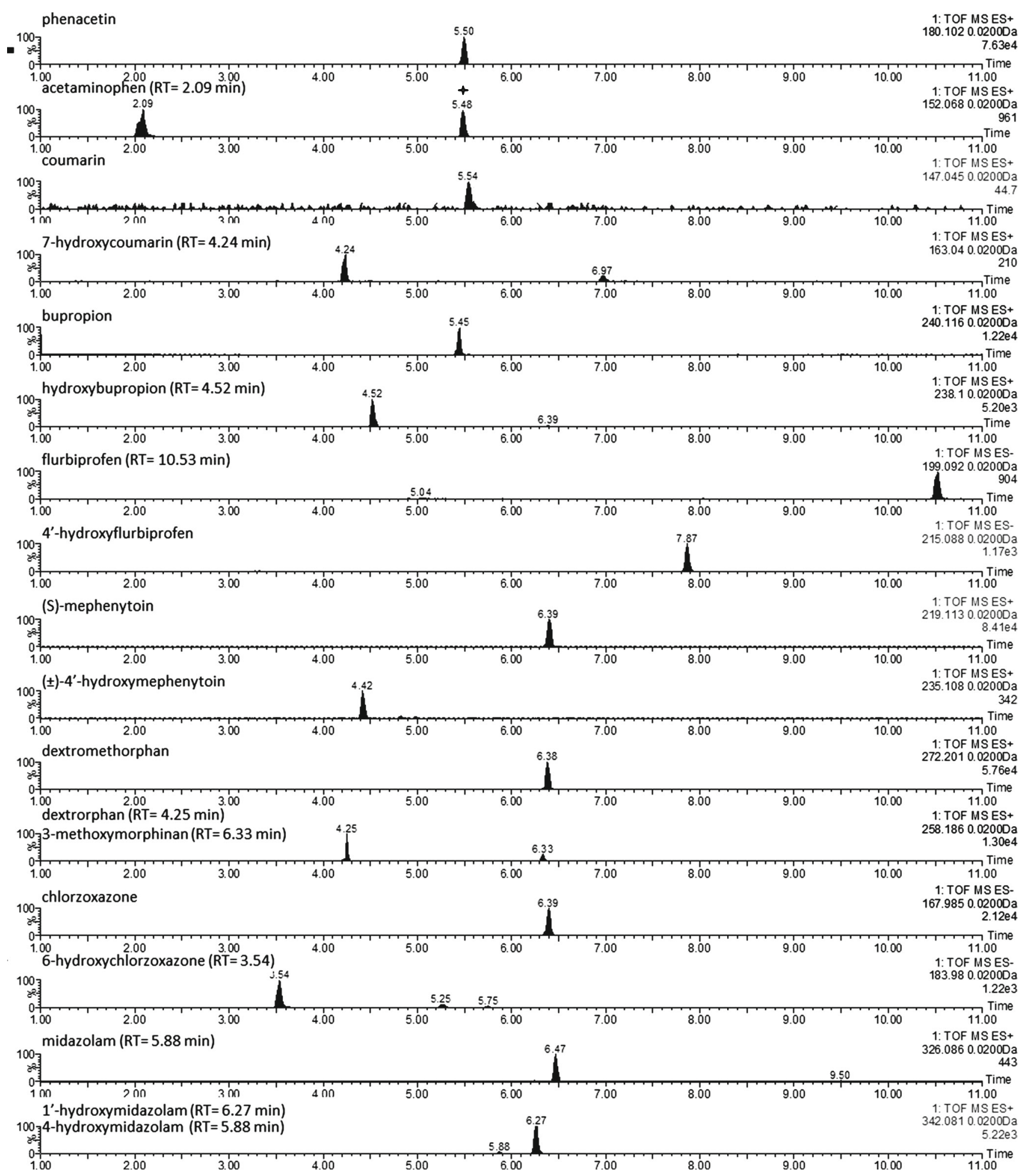

Fig. 2 Extracted ion chromatograms [of the first function mass spectrometry $(M S), \pm 0.02 \mathrm{Da}$ ] of substrates and their CYP-specific metabolites corresponding to the analysis of the cocktail microsomal incubation
(20 min). The peak highlighted with a cross results from an in-source MS fragmentation (thermal degradation) of phenacetin in conditions used for the analysis. $E S$ electrospray, $R T$ retention time, TOF time of flight reduced flow rate $(400 \mu \mathrm{L} / \mathrm{min}$ vs $600 \mu \mathrm{L} / \mathrm{min})$. Compared with the UHPLC conditions, the analysis time was increased by a factor of 3 , but the required sensitivity and an acceptable number of data points (at least seven at $4.4 \%$ of the peak height) were obtained for correct peak shape generation with both the MS function and the $\mathrm{MS}^{\mathrm{E}}$ function. 


\section{Quantitative evaluation of the method}

The selectivity of the LC-MS ${ }^{\mathrm{E}}$ method was confirmed by injecting a series of blank samples as described in detail in "Selectivity." The extracted ion chromatograms $( \pm 0.02 \mathrm{Da})$ for each analyte showed no major interferences at the corresponding retention time. Response functions were considered as linear $\left(R^{2}>0.95\right)$ over concentrations ranging from the measured lower limit of quantification to $2,000 \mathrm{ng} / \mathrm{mL}$, and the method provided the desired sensitivity for each analyte.

As shown in Table 2, the response signal (peak area) of some compounds was evaluated at three concentrations (low, medium, and high) and was slightly affected (more than $25 \%$ ) by the matrix effect, particularly for the compounds with the shortest retention times. However, this effect was corrected by the use of the metabolic ratio as the analytical response. The metabolic ratio, representing the CYP-specific metabolite to probe substrate ratio, has often been used as a parameter for in vivo and in vitro characterization of the activities of CYPs instead of exclusive monitoring of the CYP probe metabolite formation [23]. When the CYP-specific biotransformation is a major pathway of the probe substrate, the metabolite and substrate peak areas are closely correlated, and the metabolic ratio will reflect the alteration of the isoform activity (e.g., the presence of a genetic mutation).

The method precision of the $\mathrm{LC}-\mathrm{MS}^{\mathrm{E}}$ method was then estimated by triplicate injection of reconstituted mixtures of substrates and metabolites on numerous analysis days, and the variability obtained on the absolute peak area for each analyte and for the CYP-specific metabolite to the probe substrate area ratio are reported in Table 2 . Considering the analytes individually, the root mean square of the within-day variabilities of the values presented ranges from 8.3 to $28.8 \%$. The analytical variability was improved (within $\pm 20 \%$ ) for all the substrate and metabolite pairs using the absolute peak area ratio, reducing the between-day variabilities to between 4.0 and $14.5 \%$.

Variability of the microsomal incubation method for assessment of the CYP activities was then estimated on numerous days by separately incubating different aliquots of the pooled HLMs with the cocktail (freeze-thaw cycles). The metabolic variability of the incubation method for the eight CYP-specific probe substrates in pooled HLMs was within a tolerance fixed at $\pm 20 \%$. No significant batch or day effects were observed on the activities of the CYPs, except for CYP2D6 (day effect) and CYP3A (batch effect), whose variability was less than $30 \%$. Considering these results, the overall method showed the ability to generate reliable data for estimation of the relative activities of the CYPs. It has to be noted that in the case of a minor pathway, the metabolic ratio is not sufficiently sensitive to reflect the activity of the monitored CYP owing to the strong effect of substrate depletion performed by other CYPs. Because the CYP-specific biotransformations of the probe substrates selected for this cocktail were major pathways, the metabolic ratio was an appropriate value representing assessment of the in vitro activities of the CYPs without absolute quantification of the probe metabolites.

Comparison between the single-substrate and cocktail approaches

To evaluate the potential interactions among the probes of the cocktail, the CYP activities were compared using the metabolic ratios obtained for the pooled HLMs, with the individual substrates and the cocktail. For the latter, the presence of multiple probes and metabolites in the incubation sample could affect the activities of the CYPs and give an unrealistic impression of the phase I metabolic capacity of the HLMs. Considering that this type of HLM is a mixture of microsomes prepared from several donors with normal CYP phenotypic profiles, pooled HLMs possess the status of an average patient. The CYP phenotypic profiles obtained with the cocktail approach and the classic approach are reported in Fig. 3a and $b$, respectively. The measured ratios were over a range of four orders of magnitude, and only the CYPs with high enzymatic turnover (e.g., CYP2A6 and CYP3A) could be evidenced. For clarity, the activities of the CYPs obtained using the cocktail approach were considered as reference values (e.g., $100 \%$ of phase I activity). This reference profile could be represented on an octagonal radar plot (Fig. 3c) in which each axis corresponds to the relative activity of the CYP. The logarithmic scale affords equivalent visual variations in cases of increasing and respective decreasing activities of the CYPs. For example, an activity increased by a factor of 2 (i.e., $200 \%$ ) presents the same variation as that reduced by a factor of 2 (i.e., $50 \%$ ). When the two profiles are overlapped (Fig. 3d), no significant differences are observed. The activities of all of the CYPs were similar within $20 \%$, except for CYP2A6 and CYP3A. The CYP2A6 activity was decreased by approximately $38 \%$ in the cocktail assay, whereas the CYP3A activity was decreased by approximately $27 \%$, with both isoforms generating lower metabolic ratios. For these isoforms, certain interactions caused by the cocktail approach have been suggested in the literature, which were not clearly identified [20, 24-26]. Although certain CYP activities decreased owing to the inhibitory effect, the cocktail assay allowed the acquisition of similar information in a single experiment about the CYP phenotypic profile as the classic approach but using a smaller amount of microsomes and proceeding eight times faster.

Determination and alteration of the CYP phenotypic profile of allelic variant HLMs

The microsomal activities of the eight CYPs of allelic variants CYP $2 C 9^{*} 3 / * 3, C Y P 2 C 19 * 2 / * 2$, and CYP $2 D 6^{*} 4 * 4$ from 


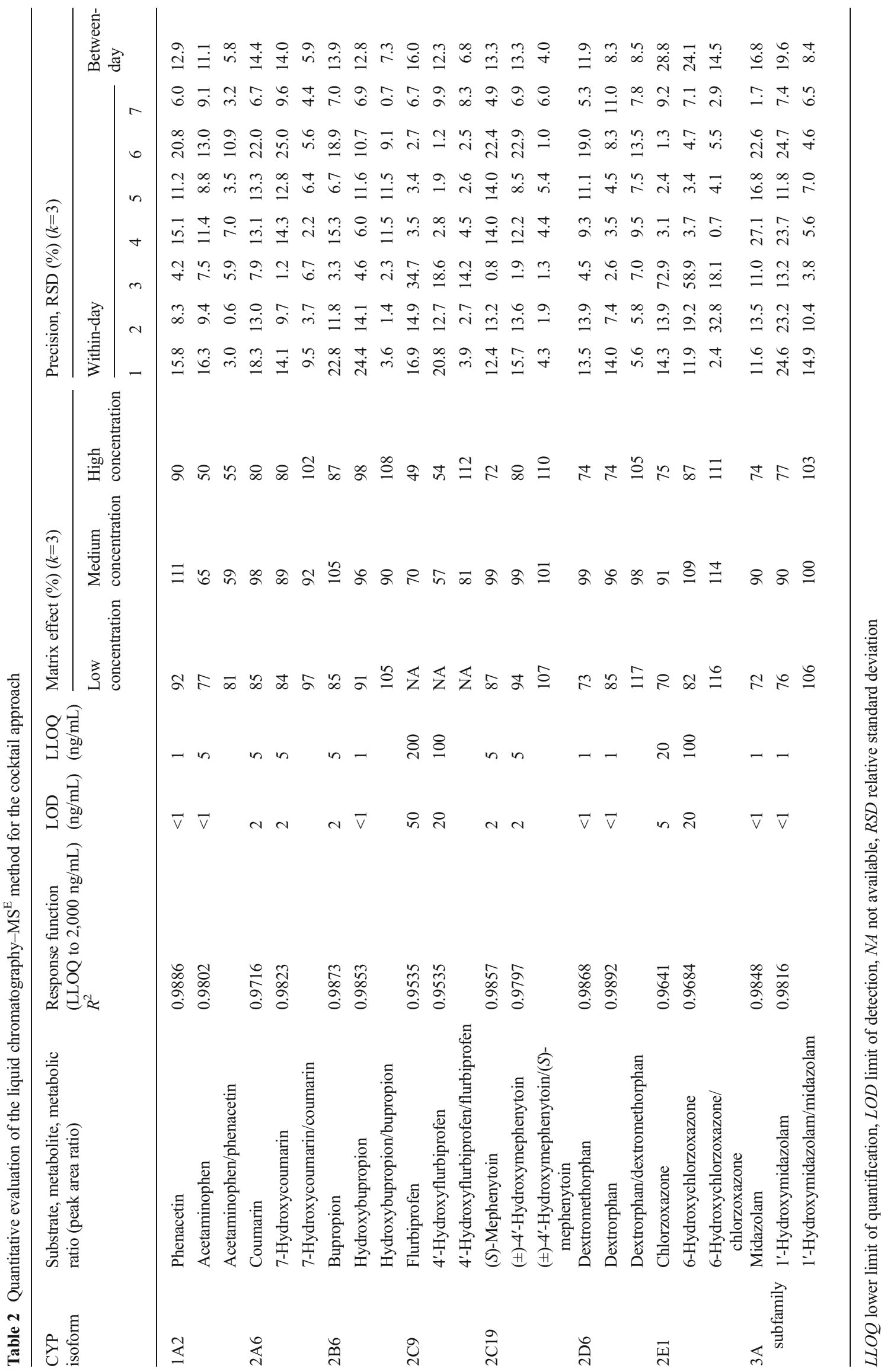


A
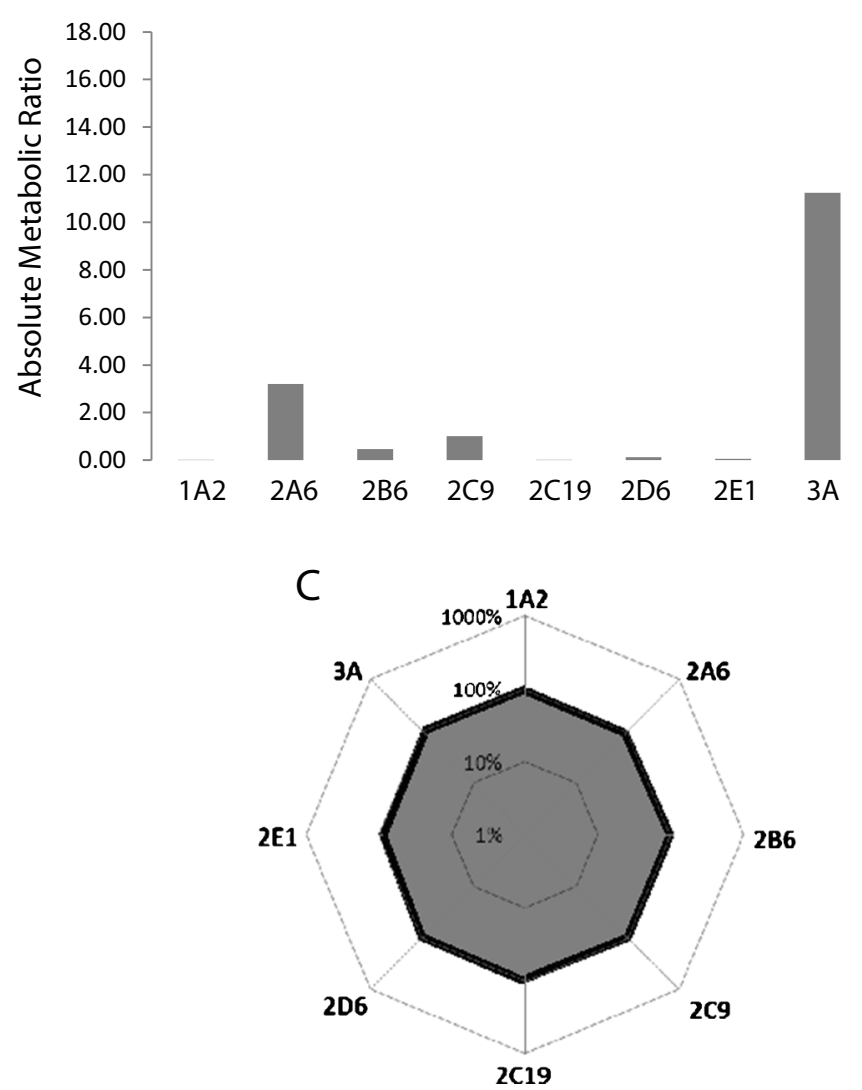

Fig. 3 Classic representation of CYP phenotypic metabolic profiles of pooled HLMs obtained with the cocktail approach (a) and with the classic approach (individual incubations of substrates) (b). Histograms showing absolute metabolic ratios for the activities of the eight CYPs. Alternative representation of CYP phenotypic profiles of pooled HLMs obtained with

single human donors were selected to be characterized with the cocktail assay developed. These HLM variants were selected because they were affected by an important genetic polymorphism, which significantly reduced the activities of three major CYPs (CYP2C9, CYP2C19, and CYP2D6) involved in the metabolism of major pharmaceutical drugs. The metabolic ratios of these single donors were expressed as the percentage of the reference value (pooled HLMs), and the normalized phenotypic profiles are reported in Fig. 4. This representation of the microsomes presents certain advantages in terms of visual interpretation. First, it demonstrates that the overall metabolic capacity of the microsomes exhibits an important interindividual variability. Then, in addition to the presence of genetic polymorphism [27], the three single-donor microsomes show three very different CYP phenotypic profiles. The results for each single-donor allelic variant HLM were in complete agreement with the genetic polymorphism and each exhibited reduced enzymatic activity for the CYP concerned compared with the activity expressed in normal pooled HLMs.
B
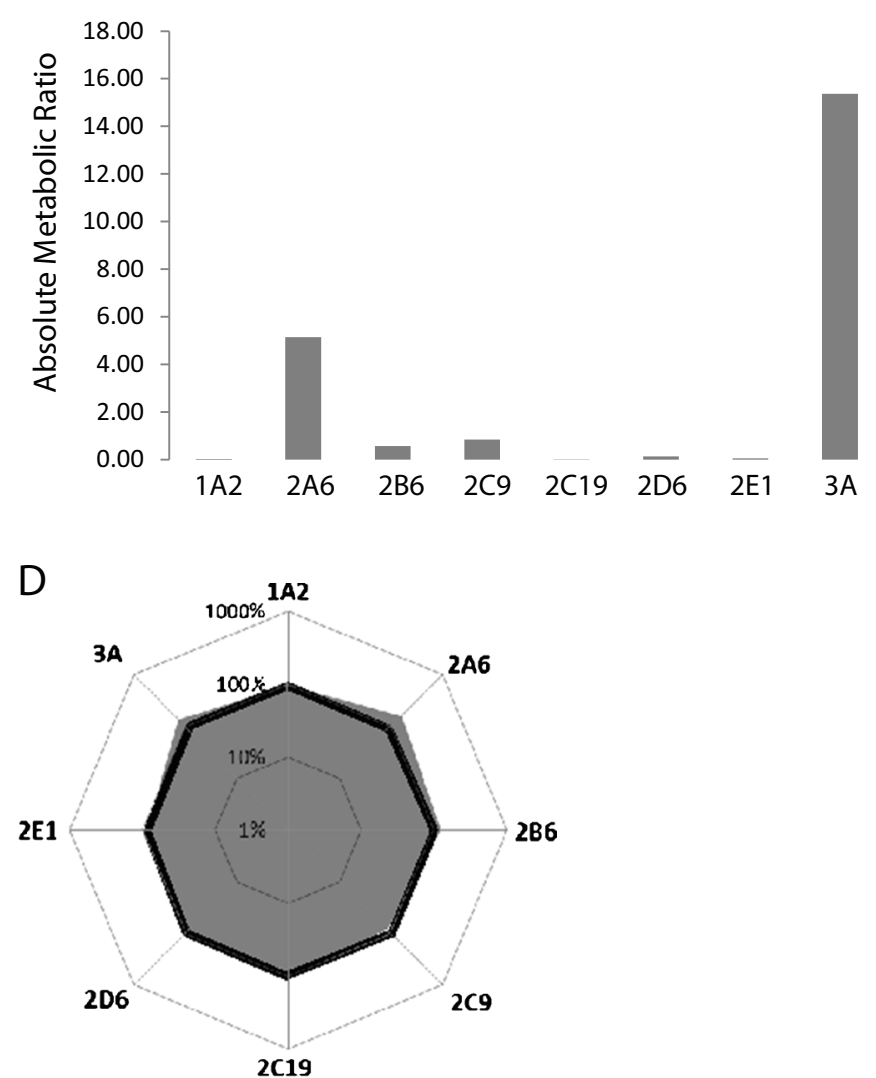

the cocktail approach (c) and with the classic approach (d). The radar chart in $\mathbf{c}$ reports the metabolic ratios expressed as the reference profile $(100 \%)$, whereas the radar chart in $\mathbf{d}$ shows the overlapping of the reference profile and the profile obtained with the classic approach expressed as a percentage of the reference

In addition to genetic variability (polymorphism), the CYP activities can be inhibited or induced by drugs. Two of the most significant enzymes participating in marketed drug metabolism, i.e., CYP2D6 and the CYP3A subfamily (especially CYP3A4) [28], are often involved in DDI.

The alteration of the activities of these CYPs and of the phenotypic profile was then studied for the allelic variant CYP2D6*4*4 HLMs. Chemical competitive inhibitors and baculosome CYP2D6 were sequentially incubated with the CYP2D6*4*4 HLMs to demonstrate the capability of the cocktail approach to show evidence of the metabolism modification. As expected and as depicted in Fig. 5a, the addition of quinidine $(1 \mu \mathrm{M})$, a CYP2D6 selective inhibitor, did not modify the overall microsomal phenotype. Owing to the very low initial activity of this allelic variant isoform (i.e., CYP2D6 was already affected by genetic polymorphism), the effect of this potent inhibitor is negligible. The specificity of this inhibitor at $1 \mu \mathrm{M}$ was confirmed by the similar profiles of the CYP activities and the noninhibited system (Fig. 4c).

Then, to improve the reduced CYP activity in the same polymorphic HLMs, this system was exposed to baculosome 
Fig. 4 Determination of CYP phenotypic profile with the cocktail approach. Application to single-donor polymorphic HLMs: a $C Y P 2 C 9^{*} 3 * * 3 ; \mathbf{b} C Y P 2 C 19 * 2 /$ $* 2$; and c CYP $2 D 6 * 4 * 4$. CYP metabolic ratios are expressed as a percentage of the metabolic ratios obtained with pooled HLMs (100 \%)

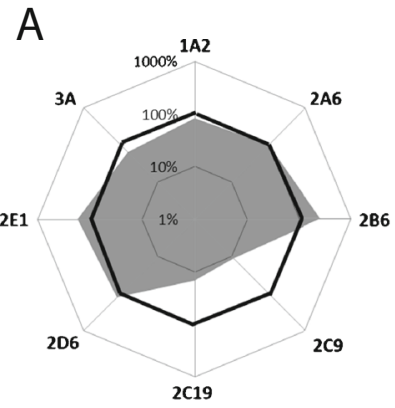

HLM with CYP2C9*3/*3

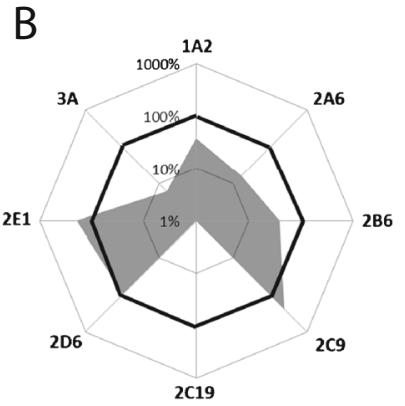

HLM with CYP2C $19 * 2 / * 2$

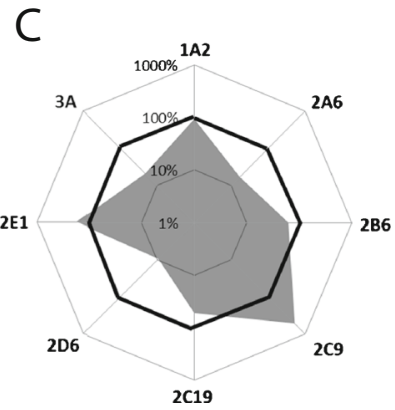

HLM with CYP2D6*4/*4

CYP2D6 (3 nM). As shown in Fig. 5b, the polymorphic HLMs recovered the CYP2D6 metabolic activity after addition of the functional isoform without modifying the overall phase I metabolic capacity.

The addition of ketoconazole, a CYP3A selective inhibitor, at a concentration of $1 \mu \mathrm{M}$ reduced the CYP3A activity, whereas the activities of the other CYPs monitored were not modified compared with the original profile (Fig. 5c), which confirms the high specificity of ketoconazole at this concentration.

QTOF data for the extension of the CYP phenotypic profile

As previously mentioned, the cocktail phase I metabolism is very complex owing to the large number of substrates and the simultaneous presence of all of the hepatic isoforms of the CYPs in the HLMs. Each substrate generates specific metabolites, but these metabolites could be substrates, creating a second generation of phase I metabolites. Therefore, the number of features to potentially monitor will increase with the number of probes in the cocktail. In this context, QTOF-MS acquisition, which does not require prior selection of the metabolites to be detected, could be advantageously applied. Indeed, known metabolites can be accurately extracted with the exact mass and narrow mass window (e.g., Fig. 2), whereas unknown metabolites can also be detected and identified using automated software (e.g., Metabolynx $\left.{ }^{\mathrm{TM}}\right)$. Two simple examples of this concept are shown in Fig. 6. In addition to 1'-hydroxylation, the CYP3A subfamily catalyzes to a minor extent the 4hydroxylation of midazolam (Fig. 6a) [29]. The resulting metabolite has the same mass as the major metabolite, but it has a different retention time, as depicted in Fig. 2. The modification of the CYP3A activity, expressed as the metabolic ratio of 4-hydroxymidazolam to midazolam in allelic variant CYP2D6*4/*4 HLMs, was in complete agreement with the alterations and was very close to that obtained for the major metabolite. As shown in Fig. 6b, the enzyme isoforms involved in the overall metabolism of dextromethorphan are CYP2D6, responsible for the O-demethylation

to dextrorphan, and CYP3A, which mediates the Ndemethylation to 3-methoxymorphinan. The specificity of CYP3A is high, but $K_{\mathrm{m}}$ for this pathway is 100 times higher than for O-demethylation, making it a minor metabolic pathway [10]. Extraction of 3-methoxymorphinan occurs at the same mass as dextrorphan, and has a retention time of 6.33 min (Fig. 2). As depicted in Fig. 6b, the modification of the CYP3A activity, expressed as the metabolic ratio of 3-methoxymorphinan to dextromethorphan in allelic variant $C Y P 2 D 6^{*} 4 * 4$ HLMs, showed results similar to those obtained considering both of the midazolam metabolites. The addition of quinidine or baculosome CYP2D6 did not affect the ratio representing this CYP3A-mediated biotransformation. As expected, the addition of ketoconazole decreased the CYP3A activity. The significantly lower affinity of dextromethorphan for CYP3A could explain the lower effect of ketoconazole on the CYP3A activity compared with midazolam.

Finally, these biotransformations could be integrated as a supplementary assessment of the CYP3A activity and, considering that each CYP isoform can potentially be involved in the formation of known and unknown metabolites from different substrates, a more precise phenotypic profile of the CYPs (e.g., "extended cocktail profile") could be generated using QTOF-MS by extracting the latent metabolic information.

\section{Conclusion}

A cocktail assay including eight probe substrates was developed to simultaneously phenotype the eight major CYPs in HLMs. The CYP activities were evaluated on the basis of the metabolic ratio measured using the $\mathrm{LC}-\mathrm{MS}^{\mathrm{E}}$ method. Separation of the analytes was performed on a $10-\mathrm{cm}$ column packed with $2.5-\mu \mathrm{m}$ particles, which primarily allowed the acquisition of a suitable chromatographic peak width compatible with the low acquisition rate generated by the $\mathrm{MS}^{\mathrm{E}}$ and pDRE association. This type of detection method made 
Fig. 5 CYP phenotypic profile of single-donor polymorphic HLMs $(C Y P 2 D 6 * 4 / 4)$ modified with $1 \mu \mathrm{M}$ quinidine (a), $3 \mathrm{nM}$ baculosome CYP2D6 (b), and $1 \mu \mathrm{M}$ ketoconazole (c), obtained with the cocktail approach. CYP metabolic ratios are expressed as a percentage of the metabolic ratios obtained with pooled HLMs (100\%)

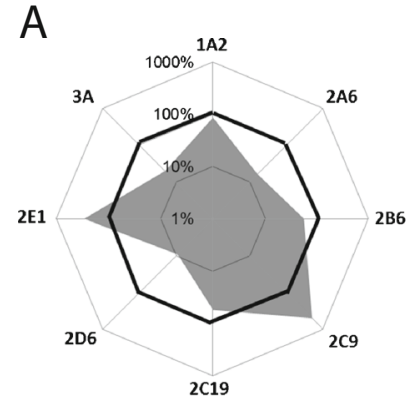

HLM with CYP2D6*4/*4 + quinidine $1 \mu \mathrm{M}$

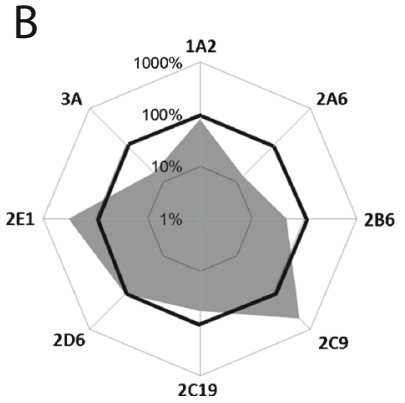

HLM with CYP2D6*4/*4 + baculosome CYP2D6 3nM

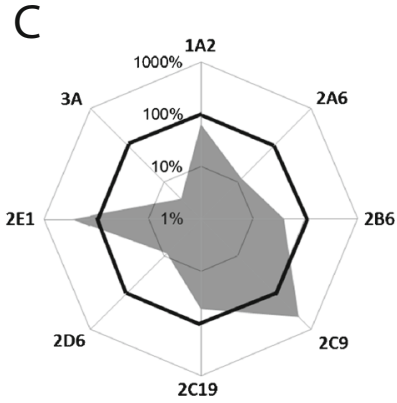

HLM with CYP2D6*4/*4 + ketoconazole $1 \mu \mathrm{M}$

possible the simultaneous acquisition of good quantitative and qualitative data with an enhanced dynamic range and improved mass accuracy measurements. The variability of the analytical method was improved using the metabolic ratio, which avoided the use of an additional analytical standard in the quenching solution. Owing to the good analytical variability, it was possible to use this parameter as an indicator of the CYP activity without performing absolute quantification.

Time-based investigations on cocktail metabolism in pooled HLMs defined $20 \mathrm{~min}$ as the optimal incubation time,

which corresponds to a compromise between linearity of metabolite formation and acceptable substrate depletion. Under these conditions, the low enzymatic turnover substrate $(S)$ mephenytoin generated a detectable amount of CYP-specific metabolite, allowing the assessment of the highly polymorphic CYP2C19 activity.

The reliability of the cocktail assay was confirmed after comparison with individual incubations of the substrates, which generated similar results. The CYP phenotypic profile resulted in the following: (1) better understanding of the

A

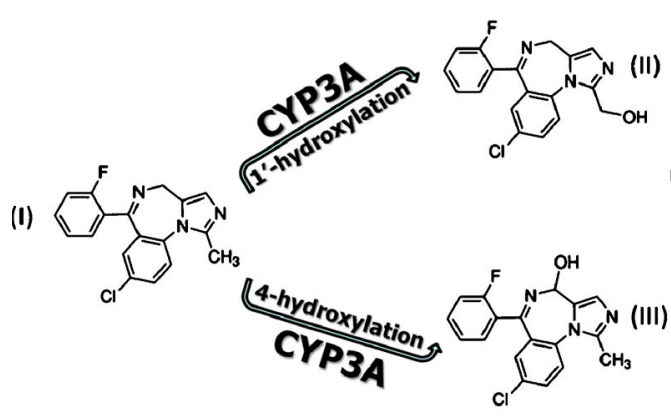

B
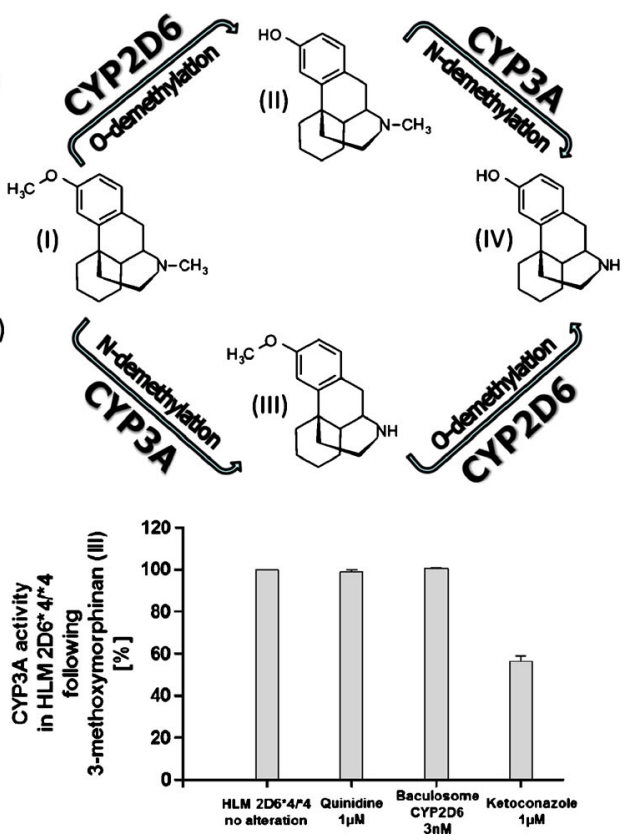

(I). The metabolites are dextrorphan (II), 3-methoxymorphinan (III), and 3-hydroxymorphinan $(\mathrm{IV})$. The histogram at the bottom represents the CYP3A activity of single-donor polymorphic HLMs $(C Y P 2 D 6 * 4 * 4)$ modified with $1 \mu \mathrm{M}$ quinidine, $3 \mathrm{nM}$ baculosome CYP2D6, or $1 \mu \mathrm{M}$ ketoconazole, using CYP3A-mediated N-demethylation of dextromethorphan to 3-methoxymorphinan. CYP metabolic ratios (3methoxymorphinan to dextromethorphan) are expressed as a percentage (mean \pm range, $n=2$ ) of the metabolic ratio obtained with no altered CYP2D6*4*4 HLMs (100\%) 
overall metabolic capacity of the microsomes tested, (2) comparison between profiles, and (3) an easily observable demonstration of the modifications of the CYP activities. Consequently, this cocktail assay was shown to be useful for phase I microsomal metabolism characterization and to rapidly highlight xenobiotics with a significant effect on the activities of CYPs, which is helpful during screening experiments. In combination with more sophisticated in vitro methods and/or with the assistance of in vitro-based computational simulation of in vivo data, this cocktail approach may be the first step toward filling the existing gap between in vitro and in vivo data.

\section{References}

1. Testa B, Pedretti A, Vistoli G (2012) Foundation review: reactions and enzymes in the metabolism of drugs and other xenobiotics. Drug Discov Today 17:549-560

2. Samer CF, Lorenzini KI, Rollason V, Daali Y, Desmeules JA (2013) Applications of CYP450 testing in the clinical setting. Mol Diagn Ther 17:165-184

3. Nicoli R, Curcio R, Rudaz S, Veuthey JL (2009) Development of an in-capillary approach to nanoscale automated in vitro cytochromes P450 assays. J Med Chem 52:2192-2195

4. Curcio R, Nicoli R, Rudaz S, Veuthey JL (2010) Evaluation of an incapillary approach for performing quantitative cytochrome P450 activity studies. Anal Bioanal Chem 398:2163-2171

5. Tolonen A, Turpeinen M, Pelkonen A (2009) Liquid chromatography-mass spectrometry in in vitro drug metabolite screening. Drug Discov Today 14:120-133

6. Nicoli R, Martel S, Rudaz S, Wolfender JL, Veuthey JL, Carrupt PA, Guillarme D (2010) Advances in LC platforms for drug discovery. Expert Opin Drug Dis 5:475-489

7. Spaggiari D, Geiser L, Daali Y, Rudaz S (2014) A cocktail approach for assessing the in vitro activity of human cytochrome CYP450s: an overview of current methodologies. J Pharm Biomed Anal. doi:10. 1016/j.jpba.2014.03.018

8. Spaggiari D, Fekete S, Eugster PJ, Veuthey JL, Geiser L, Rudaz S, Guillarme D (2013) Contribution of various types of liquid chromatography-mass spectrometry instruments to band broadening in fast analysis. J Chromatogr A 1310:45-55

9. Matuszewski BK, Constanzer ML, Chavez-Eng CM (2003) Strategies for the assessment of matrix effect in quantitative bioanalytical methods based on HPLC-MS/MS. Anal Chem 75: 3019-3030

10. Yuan R, Madani S, Wei XX, Reynolds K, Huang SM (2002) Evaluation of cytochrome $\mathrm{P} 450$ probe substrates commonly used by the pharmaceutical industry to study in vitro drug interactions. Drug Metab Dispos 30:1311-1319

11. Jerdi MC, Daali Y, Oestreicher MK, Cherkaoui S, Dayer P (2004) A simplified analytical method for a phenotyping cocktail of major CYP450 biotransformation routes. J Pharm Biomed Anal 35:12031212

12. Bosilkovska M, Déglon J, Samer C, Walder B, Desmeules J, Staub C, Daali Y (2014) Simultaneous LC-MS/MS quantification of Pglycoprotein and cytochrome $\mathrm{P} 450$ probe substrates and their metabolites in DBS and plasma. Bioanalysis 6:151-164

13. Watanabe A, Nakamura K, Okudaira N, Okazaki O, Sudo KI (2007) Risk assessment for drug-drug interaction caused by metabolismbased inhibition of CYP3A using automated in vitro assay systems and its application in the early drug discovery process. Drug Metab Dispos 35:1232-1238

14. Sevrioukova IF, Poulos TL (2013) Understanding the mechanism of cytochrome P450 3A4: recent advances and remaining problems. Dalton Trans 42:3116-3126

15. Tucker GT, Houston JB, Huang SM (2001) Optimizing drug development: strategies to assess drug metabolism/transporter interaction potential - towards a consensus. Br J Clin Pharmacol 52:107-117

16. Bjornsson TD, Callaghan JT, Einolf HJ, Fischer V, Gan L, Grimm S, Kao J, King SP, Miwa G, Ni L, Kumar G, McLeod J, Obach RS, Roberts S, Roe A, Shah A, Snikeris F, Sullivan JT, Tweedie D, Vega JM, Walsh J, Wrighton SA (2003) The conduct of in vitro and in vivo drug-drug interaction studies: a Pharmaceutical Research and Manufacturers of America (PhRMA) perspective. Drug Metab Dispos 31:815-832

17. Di L, Kerns EH, Li SQ, Carter GT (2007) Comparison of cytochrome P450 inhibition assays for drug discovery using human liver microsomes with LC-MS, rhCYP450 isozymes with fluorescence, and double cocktail with LC-MS. Int J Pharm 335:1-11

18. Walsky RL, Obach RS (2004) Validated assays for human cytochrome P450 activities. Drug Metab Dispos 32:647-660

19. Weaver R, Graham KS, Beattie IG, Riley RJ (2003) Cytochrome P450 inhibition using recombinant proteins and mass spectrometry/ multiple reaction monitoring technology in a cassette incubation. Drug Metab Dispos 31:955-966

20. Kozakai K, Yamada Y, Oshikata M, Kawase T, Suzuki E, Haramaki Y, Taniguchi H (2012) Reliable high-throughput method for inhibition assay of 8 cytochrome $\mathrm{P} 450$ isoforms using cocktail of probe substrates and stable isotope-labeled internal standards. Drug Metab Pharmacokinet 27:520-529

21. US Food and Drug Administration (2011) Drug development and drug interactions: table of substrates, inhibitors and inducers. http:// www.fda.gov/drugs/developmentapprovalprocess/ developmentresources/druginteractionslabeling/ucm093664.htm. Accessed Jan 2014

22. Chauret N, Gauthier A, Nicoll-Griffith DA (1998) Effect of common organic solvents on in vitro cytochrome P450-mediated metabolic activities in human liver microsomes. Drug Metab Dispos 26:1-4

23. Baudoin R, Prot JM, Nicolas G, Brocheton J, Brochot C, Legallais C, Benech H, Leclerc E (2013) Evaluation of seven drug metabolisms and clearances by cryopreserved human primary hepatocytes cultivated in microfluidic biochips. Xenobiotica 43:140-152

24. Palmer JL, Scott RJ, Gibson A, Dickins M, Pleasance S (2001) An interaction between the cytochrome $\mathrm{P} 450$ probe substrates chlorzoxazone (CYP2E1) and midazolam (CYP3A). Br J Clin Pharmacol 52:555-561

25. Pillai VC, Strom SC, Caritis SN, Venkataramanan R (2013) A sensitive and specific CYP cocktail assay for the simultaneous assessment of human cytochrome $\mathrm{P} 450$ activities in primary cultures of human hepatocytes using LC-MS/MS. J Pharm Biomed Anal 74: $126-132$

26. Dierks EA, Stams KR, Lim HK, Cornelius G, Zhang HL, Ball SE (2001) A method for the simultaneous evaluation of the activities of seven major human drug-metabolizing cytochrome P450s using an in vitro cocktail of probe substrates and fast gradient liquid chromatography tandem mass spectrometry. Drug Metab Dispos 29:23-29

27. Wilson ZE, Rostami-Hodjegan A, Burn JL, Tooley A, Boyle J, Ellis SW, Tucker GT (2003) Inter-individual variability in levels of human microsomal protein and hepatocellularity per gram of liver. Br J Clin Pharmacol 56:433-440

28. Guengerich FP (2008) Cytochrome P450 and chemical toxicology. Chem Res Toxicol 21:70-83

29. Patki KC, von Moltke LL, Greenblatt DJ (2003) In vitro metabolism of midazolam, triazolam, nifedipine, and testosterone by human liver microsomes and recombinant cytochromes P450: role of CYP3A4 and CYP3A5. Drug Metab Dispos 31:938-944 